\title{
Inverter Control Performance Study on Solar PV Systems and Its Effect on Power Quality
}

\author{
Vandana Tiwari \\ M. Tech Scholar \\ Truba Institute of Engineering and \\ Information Technology \\ vandanatiwari98@gmail.com
}

\author{
Dr. Rajeev Arya \\ Director \\ Truba Institute of Engineering and \\ Information Technology \\ $\underline{\text { Rajeev.arya@trubainstitute.ac.in }}$
}

\author{
Shravan vishwakarma \\ Professor \\ Truba Institute of Engineering and \\ Information Technology \\ shravanmits@gmail.com
}

\begin{abstract}
Power generation faces a major challenge in meeting peak load requirements. Energy suppliers are highly dependent on fossil fuels due to the limited resources of non-renewable energy production. Therefore, researchers and scientists are looking for distributed generators (DGs) to provide additional power during peak periods of the energy curve. Solar energy gives them an extra twist to meet the load demand during this time. As a result, the grid-based solar photovoltaic (PV) system is attracting particular attention from researchers and industry in order to reduce the burden of fossil fuel power generation. Single-stage and two-stage photovoltaic systems are suitable for grid connection with or without battery holder. This article provides a comprehensive overview of a grid connected solar system. The entire architecture of the on-grid photovoltaic system includes the construction of a photovoltaic generator, MPPT methods and DC-DC converters.
\end{abstract}

Keywords: DC, PV System, Distributed Generators, Maximum Power Point Tracking.

\section{INTRODUCTION}

Solar power is the most abundant and cleanest renewable energy source in the world and is technically ready for a variety of uses, e.g. B. generate electricity for private, commercial or industrial consumption. Due to the reduced cost of photovoltaic (PV) modules and the further development of the conventional power converter from the point of view of efficiency, reliability and cost, it has become an essential part of modern energy systems. Power electronics technology has become an important element of decentralized generation (DG) and facilitates the integration of renewable energy sources into the grid [1].

Photovoltaic converters are often used to convert the DC voltage generated by the PV generator into AC voltage. Two-step power conversion is currently the most common approach to manage the $\mathrm{DC}$ voltage range generated by the PV module. This configuration consists of a DC-DC converter which, in combination with the MPP (Maximum Power Point Tracking) algorithms, performs a MPPT (Maximum Power Point Tracking) and a DC / AC converter stage to power the grid or the grid and local charges. Well known control approaches such as Perturb and Observe, INC (Incremental Conductance), CV (Constant Voltage) algorithms and improved versions thereof have been developed to monitor MPP operation. Multi-stage power conversion systems reduce the overall energy efficiency and reliability of the photovoltaic system and increase costs [2]. Therefore, from the point of view of efficiency and reliability, some publications have reported on single-stage photovoltaic systems connected to the grid consisting of a photovoltaic generator and DC-AC converter with MPPT for the control of active and reactive power using linear control techniques conventional. Most of the studies have been developed for three-phase systems, which are widely used in photovoltaic systems. Due to the high penetration of low-power single-phase photovoltaic systems, some researchers in recent years have focused their studies on single-phase systems not only from the point of view of efficiency and reliability, but also for grid support. Providing grid support can reduce the negative effects that can lead to disconnection of the PV system from the grid in the event of grid failure, as required by current grid standards in some countries. He says PV systems are expected to become more dynamic in the near future with features such as low voltage throughput (LVRT) and grid support capacity. To date, however, some countries' current grid standards are still in place and should be revised to allow these additional functions for photovoltaic systems [3] [4].

Due to the inherent characteristics of the PV cell and the non-linear switching functions of the inverter, PV systems exhibit non-linear behavior that can adversely affect 
system performance if not properly compensated. Since nonlinear controls directly address nonlinearities, there are advanced and effective nonlinear control techniques and schemes in the literature that are applied to grid PV systems. Techniques such as recoil control, slip control and feedback linearization were introduced. Most of these studies refer to the two-level topology for three-phase or single-phase systems.[5]

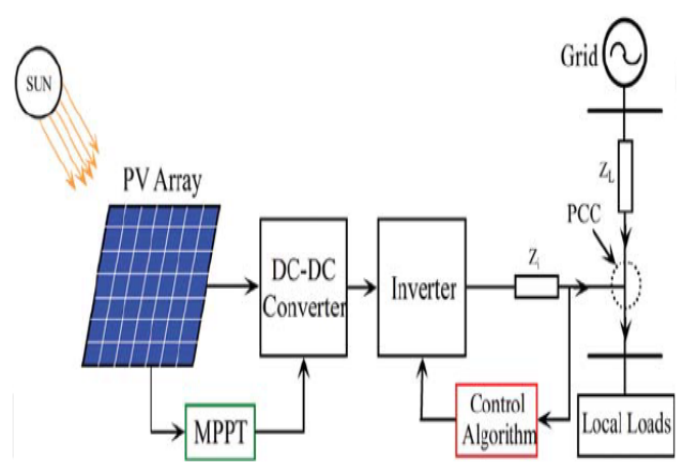

Fig. 1 Grid-Interfaced Solar Photovoltaic System

\section{LiterATURE REVIEW}

S. M. Ashabani et al. [6] The energy sector is entering the era of decentralized production (DG) and microgrids (MG). However, the stability and operational aspects of converter-dominated DG MGs face many challenges. Among these, the most important are: 1) lack of physical inertia; 2) comparable dimensions of the converters; 3 ) reciprocal interactions between generators; 4) delays in detecting islets; and 5) sudden large disturbances associated with island mode transition, grid recovery and load power changes. To overcome these difficulties, a new control topology based on important signals for DG converters is presented in this document, suitable for both grid and stand-alone operation modes without the need to reconfigure the control system and without detection.

Q.-C. Zhong et al. [7] This article takes a drastic step to improve the synchronous converter as a self-synchronized synchronous slip by removing the dedicated synchronization unit. It can automatically synchronize with the network before connection and track the network frequency after connection. This greatly improves performance, reduces the complexity and computing effort of the controller. All original synchronous converter functions such as frequency and voltage control, active power and reactive power control are maintained.
T.-F. Wu et al. [8] This article presents a D- $\Sigma$ (Division Summation) digital control for a three-phase inverter, with which active and reactive power can be fed into the AC grid. The proposed D- $\Sigma$ approach summarizes the fluctuations of the inductance current over a switching cycle in order to directly derive control laws, with which it is possible to overcome the limitation of the $\mathrm{d}$-q transformation. The inverter with this control can drive or delay various power factors (PFs) allowing for large fluctuations in filter inductance and line voltage distortion and greatly reducing core size.

W. R. Issa et al. [9]This article examines the transient circulation power between inverters connected in parallel during accidental isolation and proposes a regulation to limit it. The controller monitors the intermediate circuit voltage and sets the power set point proportional to the voltage rise. A small-signal model of an island microarray is developed and used to design the controller. The simulation and test results are presented to validate the design.

J. H. Teng et al. [10] This article provides an MPPT-based parameter estimate for PVG. Based on the measured voltages and currents and the characteristic output function of the PVG, the proposed MPPT uses parameter estimation to directly calculate the solar radiation and temperature. The proposed MPPT can effectively reduce the maximum performance tracking time of conventional MPPT methods and increase the energy recovered during MPPT. The simulation and experimental results comparing the proposed MPPT with a P\&O MPPT show the performance obtained by the proposed MPPT.

\section{PV ARRAY}

The power generated by a single photovoltaic cell is very low. For high power applications or grid-related conditions, the output power and voltage are generated by a series of parallel groups and combinations of PV cells. These combinations are called PV modules and form PV modules. These panels are used to form the desired PV generator. Several topologies are used in the literature for the formation of integrated photovoltaic systems (BIPS) and rooftop photovoltaic systems (RTPS) [11]. Different configurations of photovoltaic panels proposed by the researchers are illustrated. The series and parallel topologies have some disadvantages such as lower current or lower voltage. The series to parallel (SP) configuration is suggested by first connecting the modules in series, then 
connecting the strings in parallel to obtain the required voltage and current. A TCT (Total Cross Tied) configuration is proposed to obtain equal voltages on the different connections and the sum of the currents on the connections. In the bridge configuration, a bridge is formed by connecting two modules in series and two in parallel to obtain the added value of voltage and currents. The Honey Comb (HC) configuration is only a modification of the BL configuration to take advantage of both TCT and BL configurations [12].

\section{Maximum Power Point Tracking}

The overall efficiency of the PV system can be improved by running the system on MPP. Monitoring the maximum power point on the PV curve, regardless of environmental changes such as temperature and irradiation, is called maximum power point monitoring. An appropriate MPPT method is required to keep the operating point at the point of maximum power so that maximum power can be drawn from the solar PV system.

\section{A. Basics of MPPT}

MPPT techniques are used to extract maximum performance regardless of environmental changes. In general, there are two options for getting the maximum power from the photovoltaic system: mechanical tracking and electrical tracking. Mechanical tracking is considered physical tracking. During this process, the alignment of the PV system is changed based on changes in the position of the sun at a specified angle. Changes can also be made for seasons of an entire year.

Orientation changes are made using a manual exercise or with an automatic speed setting. In electrical tracing, the $\mathrm{I}-\mathrm{V}$ curve is plotted using a series of algorithms. The PV parameters (voltage, current, temperature and irradiation) are saved and entered into the algorithm according to your needs. The required MPPT technology calculates reference values for various parameters. The calculated reference values are compared with the values before the end of the converter. Then the current power at the MPP is compared with the measured value. The differences between these values are minimized by changing the duty cycle of the converter. A PI controller or a hysteresis controller is generally used to set the duty cycle of the converters. In some cases, however, the duty cycle is determined directly using various algorithms.

\section{DC-DC CONVERTER}

The DC-DC converter is used to increase or decrease the PV output voltage as needed. The operation of DC-DC converters fulfills two important tasks: the integration of PV or renewable sources into the grid and the detection of MPP on the I-V curve using suitable MPPT control algorithms. Ideally, switching converters are 100\% efficient, but in practical applications the efficiency is reduced due to some conduction and switching losses. The voltage waveform has a switching pattern and must be filtered using capacitors and inductors. Therefore, switched DC / DC converters have at least one power switch with a capacitor and an inductor. Many DC-DC converter circuit topologies have already been published in the literature. DC-DC converters fall into two main categories: isolated converters and non-isolated converters. Using some electrical barriers, an isolated converter provides some isolation between the input side circuit and the output side circuit. Isolation can be useful when designing multiple output ports with different voltage levels.

An electrical transformer is usually issued for isolation purposes. It is also possible to achieve gain and attenuation. By changing the transformation ratio of the transformer. Galvanic isolation can suppress electromagnetic interference between the source and the load. These converters are considered an extension of the basic converters, but the cost and size of isolated converters are very high due to the transformer and additional stage of the DC-DC converter. They are useful in some special applications where isolationism is required. There is no isolation transformer in non-isolated converters. They are useful in low stress elevation conditions. Therefore, they are implemented for applications such as water pumping systems, stand-alone photovoltaic systems, etc. For safety reasons, isolated converters are generally preferred for grid connected PV systems. There are four topologies of DC-DC converters commonly used in solar photovoltaic systems. You can work in two different modes, the continuous conduction mode (CCM) or the discontinuous conduction mode (DCM). the performance is analyzed using the following parameters.

(i) Voltage Gain (Av)

(ii) Current Gain $\left(\mathrm{A}_{\mathrm{i}}\right)$

(iii) Input Impedance $\left(\mathrm{R}_{\mathrm{i}}\right)$

(iv) Critical Inductance ( $\left.\mathrm{L}_{\text {crit }}\right)$ 
(v)

\section{Critical Capacitance ( $\left.\mathrm{C}_{\text {crit }}\right)$ \\ VI. Shunt COMPENSATION}

The shunt compensation system can basically be developed with a voltage source, nonlinear load, and shunt connected power system.

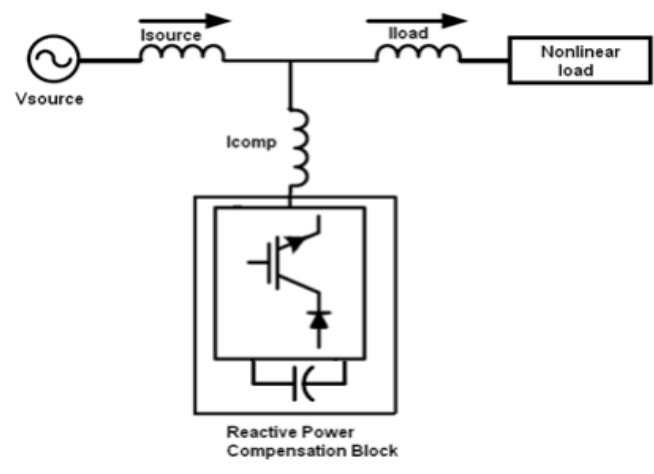

Fig. 2 Parallel (shunt) connection

In this system, when the non-linear load is an inductive load, the power system needs reactive power for precise operation, so this requirement must be provided by the voltage source. By connecting the branch system, the compensation current (Icomp) of the opposite direction is injected with equivalent amplitude into the system, and then the reactive current of the load is compensated, the voltage regulation is improved and the unity of the power factor is obtained.

\section{VII.SERIES COMPENSATION}

The series compensation system can in principle also be developed with a voltage source, a non-linear load and a series-connected power supply system.

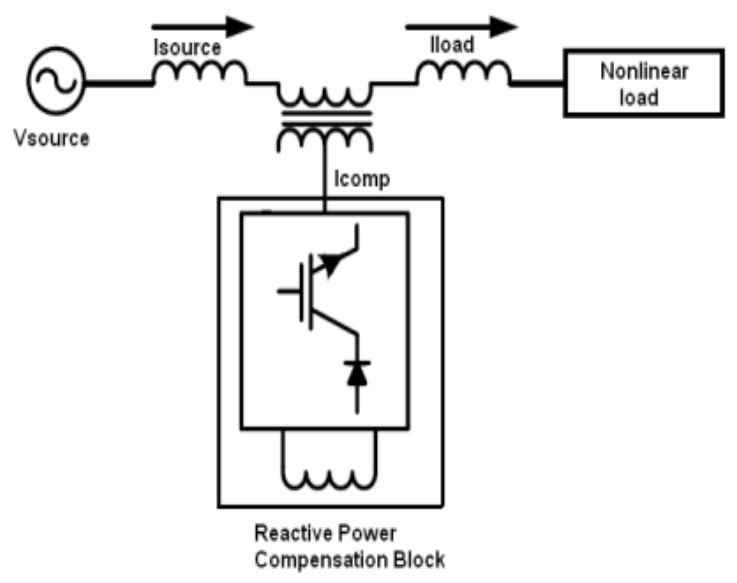

Fig. 3 Series connection

\section{Issues CAused In GRID-ConNected PV SYSTEMS}

As the total cost of installing and owning GCPVS systems decreases, the adoption of this technology in the residential, commercial and utility sectors increases. Although GCPVS has many advantages such as: for example, long life (25-30 years), low operating and maintenance costs, and obvious environmental advantages over fossil fuel power plants, GCPVS has its challenges. Numerous scientific articles have shown that the massive adoption and spread of GCPVS could put enormous pressure on the power grid.

\section{CONCLUSION}

Photovoltaic systems have become a mature technology for generating electricity. However, they introduce many negative effects into power grids. Although the solar PV market has seen astronomical growth and cost reductions in recent years, there are many technical challenges and economic realities that need to be balanced for GD's resources such as GCPVS to be on par with conventional generation. For successful mass adoption of GCPVs, new technologies need to be developed to enable the inverter to do more than just $\mathrm{DC}$ to $\mathrm{AC}$ conversions. This article provides an overview of a photovoltaic generator and MPPT technology. The entire architecture of the grid interface solar PV system includes construction of a PV generator, MPPT methods, DC-DC converters and compression shunts.

\section{REFERENCES}

[1] YeqinWang, BeibeiRen "Bounded-voltage Power Flow Control for Grid-tied PV Systems" Volume 50, Issue 1, July 2017, Pages 7699-7704

[2] Beibei Ren, Yeqin Wang "Fault Ride-Through Enhancement for Grid-Tied PV Systems With Robust Control” Volume: 65 , Issue: 3 ,August 2017

[3] Deo, S., Jain, C., and Singh, B. "A PLL-less scheme for singlephase grid interfaced load compensating solar PV generation system". IEEE Trans. Ind. Informat., 11(3), 692- 699 (2015).

[4] Qing-Chang Zhong, Beibei Ren "Robust Power Flow Control of Grid-Connected Inverters" Volume: 63 , Issue: 11 , Nov. 2016

[5] Wang et al., Y. Wang, B. Ren, Q.C. Zhong "Robust control of dcdc boost converters for solar systems". In Proc. American Control Conference, to be published (2017).

[6] S. M. Ashabani and Y. A.-R. I. Mohamed. A flexible control strategy for grid-connected and islanded microgrids with enhanced stability using nonlinear microgrid stabilizer. IEEE Trans. Smart Grid,3(3):1291-1301, Sept. 2012.

[7] Q.-C. Zhong, P.-L. Nguyen, Z. Ma, and W. Sheng. Selfsynchronized synchronverters: Inverters without a dedicated synchronization unit. IEEE Trans. Power Electron., 29(2):617630, Feb. 2014. 
[8] T.-F. Wu, C.-H. Chang, L.-C. Lin, G.-R. Yu, and Y.-R. Chang. A $\mathrm{D}-\Sigma$ digital control for three-phase inverter to achieve active and reactive power injection. IEEE Trans. Ind. Electron., 61(8):38793890, Aug.2014.

[9] W. R. Issa, M. A. Abusara, and S. M. Sharkh. Control of transient power during unintentional islanding of microgrids. IEEE Trans.PowerElectron., 30(8):4573-4584, Aug. 2015.

[10] J. H. Teng, W. H. Huang, T. A. Hsu and C. Y. Wang, "Novel and fast maximum power point tracking for photovoltaic generation", IEEE Trans. Ind. Electron., vol. 63, no. 8, pp. 4955-4966, Aug. 2016.

[11] Manna, D. L., Vigni, V. L., Sanseverino, E. R., Dio, V. D., andRomano, P. (2014). Reconfigurable electrical interconnection strategiesfor photovoltaic arrays: a review. Renew. Sustain. Energy Rev. 33,412-426.

[12] Ramaprabha, R., and Mathur, B. L. (2012). A comprehensive reviewand analysis of solar photovoltaic array configurations under partialshaded conditions. Int. J. Photo Energy. 2012. 